\title{
COVID-19: Um desafio epidemiológico
}

\author{
COVID-19: An epidemiological challenge \\ COVID-19: Un desafío epidemiológico
}

Recebido: 20/05/2021 | Revisado: 29/06/2021 | Aceito: 09/06/2021 | Publicado: 23/06/2021

\author{
Rosilene Ferreira Cardoso \\ ORCID: https://orcid.org/0000-0002-5834-8443 \\ Universidade Federal do Amapá, Brasil \\ E-mail: rosilenecardoso7@gmail.com \\ Diovana de Sena Alberto \\ ORCID: https://orcid.org/0000-0002-6983-2791 \\ Centro de Informação e Análise da Situação de Saúde, Brasil \\ E-mail: diogemeas@gmail.com \\ Silvia Cláudia Cunha Maués \\ ORCID: https://orcid.org/0000-0001-8404-3574 \\ Centro de Informação e Análise da Situação de Saúde, Brasil \\ E-mail: silvia-edi@hotmail.com \\ Sandro Rogério Mendes da Silva \\ ORCID: https://orcid.org/0000-0002-7888-3443 \\ Centro de Informação e Análise da Situação de Saúde, Brasil \\ E-mail: Sandro.sifilis@gmail.com \\ Adalton da Costa Abreu \\ ORCID: https://orcid.org/0000-0003-0107-9104 \\ Centro de Informação e Análise da Situação de Saúde, Brasil \\ E-mail: adaltoabreu79@gmail.com \\ Jucileine dos Santos Machado Coelho \\ ORCID: https://orcid.org/0000-0002-6411-7924 \\ Centro de Informação e Análise da Situação de Saúde, Brasil \\ E-mail: jsmc.ap@hotmail.com \\ Maricélia Tavares Barbosa Marinho \\ ORCID: https://orcid.org/0000-0001-9950-4492 \\ Centro de Informação e Análise da Situação de Saúde, Brasil \\ E-mail: maricelia.flp@gmail.com \\ Iracilda Costa da Silva Pinto \\ ORCID: https://orcid.org/0000-0002-7717-2252 \\ Centro de Informação e Análise da Situação de Saúde, Brasil \\ E-mail: ics_pinto@hotmail.com \\ Antônio Alexandre Valente Meireles \\ ORCID: https://orcid.org/0000-0002-4646-5079 \\ Universidade Federal do Amapá, Brasil \\ E-mail: alevalente14@gmail.com
}

\section{Resumo}

Objetivo: analisar o comportamento da letalidade e mortalidade e o perfil de desenvolvimento da COVID-19 nos estados de maior risco de adoecimento até 15 de março de 2021. Metodologia: Estudo descritivo, retrospectivo e analítico dos dados epidemiológicos dos estados da faixa vermelha de risco de adoecimento até a data de 15 de março de 2021. Na análise, priorizou-se 04 municípios com maior incidência em cada estado. Resultados e Discussão: Maior contribuição de casos confirmados observado na região Sudeste, Nordeste, Centro Oeste e Norte, respectivamente. Dos 13 estados, 07 deles em apenas 04 municípios contribuíram com mais de 40\% de casos confirmados. As taxas de mortalidade variaram de 0,07 (Maranhão) a 1,08 (Pará) e as taxas de letalidade entre os estados variaram de 1,34 (Amapá e Tocantins) e 2,90 (São Paulo). Nos estados com maior concentração de casos confirmados em menor número de municípios, existe a possibilidade de bloqueio da doença, através de ações especificas, enquanto nos estados em que disseminação do vírus é observada em vários municípios, a tendência de descontrole é evidente. Conclusão: Estratégias de controle sugeridas: educação para a saúde; uso de máscara obrigatória, participação dos educadores do ensino público e privado na educação sanitária junto ás famílias de seus alunos; barreiras sanitárias nas rodovias dos municípios que juntos estejam acima de $40 \%$ de casos confirmados, uso da inteligência local em saúde, que combina o rastreamento de contatos e testes rápidos para mitigar surtos de COVID-19, por todos os gestores das três esferas de governo e avaliações periódicas do quadro epidemiológico, para que as estratégias implementadas continuem ou sejam aperfeiçoadas.

Palavras-chave: COVID-19; SARS-CoV2; Infecções por Coronavírus; Vigilância em saúde pública; Pandemia. 


\begin{abstract}
Objective: to analyze the development profile of COVID 19 in the states most at risk of illness until March 15, 2021; calculation of the COVID 19 lethality and mortality rates among the highest risk states. Methodology: Descriptive, retrospective and analytical study of the epidemiological data of the states of the red belt at risk of illness until the date of March 15, 2021. In the analysis, we prioritized 04 municipalities with the highest incidence in each state. Results and Discussion: Higher contribution of confirmed cases observed in the Southeast, Northeast, Midwest and North regions, respectively. Of the 13 states, 07 of them in only 04 municipalities contributed with more than $40 \%$ of confirmed cases. Mortality rates ranged from 0.07 (Maranhão) to 1.08 (Pará) and lethality rates between states ranged from 1.34 (Amapá and Tocantins) and 2.90 (São Paulo). In the states with the highest concentration of confirmed cases in a smaller number of municipalities, there is the possibility of blocking the disease, through specific actions, and in the states dissemination of the Sars-Cov-2 virus in several municipalities, the tendency to lose control is evident. Conclusion: Suggested control strategies: health education; use of mandatory mask, participation of public and private educators in health education with the families of their students; sanitary barriers on the highways of municipalities that together are above $40 \%$ of confirmed cases, use of local health intelligence, which combines contact tracking and rapid tests to mitigate outbreaks of COVID-19, by all managers of the three spheres of government and periodic assessments of the epidemiological picture, so that the strategies implemented continue or are improved.
\end{abstract}

Keywords: COVID-19; SARS-CoV-2; Coronavirus infection; Public health surveillance; Pandemic.

\begin{abstract}
Resumen
Objetivo: analizar el perfil de desarrollo de COVID 19 en los estados con mayor riesgo de enfermedad hasta el 15 de marzo de 2021; cálculo de las tasas de letalidad y mortalidad de COVID 19 entre los estados de mayor riesgo. Metodología: Estudio descriptivo, retrospectivo y analítico de los datos epidemiológicos de los estados del cinturón rojo en riesgo de enfermedad hasta la fecha del 15 de marzo de 2021. En el análisis priorizamos 04 municipios con mayor incidencia en cada estado. Resultados y Discusión: Mayor contribución de casos confirmados observada en las regiones Sudeste, Nordeste, Medio Oeste y Norte, respectivamente. De los 13 estados, 07 de ellos en solo 04 municipios contribuyeron con más del $40 \%$ de los casos confirmados. Las tasas de mortalidad oscilaron entre 0,07 (Maranhão) y 1,08 (Pará) y las tasas de letalidad entre los estados oscilaron entre 1,34 (Amapá y Tocantins) y 2,90 (São Paulo). En los estados con mayor concentración de casos confirmados en un menor número de municipios, existe la posibilidad de bloquear la enfermedad, a través de acciones específicas y en los estados la propagación del virus Sars-Cov-2 en varios municipios, la tendencia a perder el control es evidente. Conclusión: Estrategias de control sugeridas: educación para la salud; uso de mascarilla obligatoria, participación de educadores públicos y privados en educación para la salud con las familias de sus alumnos; barreras sanitarias en las carreteras de los municipios que en conjunto están por encima del $40 \%$ de los casos confirmados, uso de inteligencia sanitaria local, que combina seguimiento de contactos y pruebas rápidas para mitigar brotes de COVID-19, por parte de todos los gestores de las tres esferas de gobierno y evaluaciones periódicas del cuadro epidemiológico, para que las estrategias implementadas continúen o mejoren.
\end{abstract}

Palabras clave: COVID-19; SARS-CoV-2; Infecciones por Coronavirus; Vigilancia em salud pública; Pandemia.

\title{
1. Introdução
}

Desde dezembro de 2019, o mundo se deparou com uma nova doença, agressiva e misteriosa, emergida na cidade de Wuhan, localizada na província de Hubei, na China, que se disseminou para todo o globo. Uma nova espécie de coronavírus, cuja doença recebeu o nome de COVID-19, que se tornou uma emergência de relevância internacional (Zhu et al, 2019; Li et al, 2020; Houshue et al, 2020; Koven, 2020). A infecção por SARS-CoV-2 acarreta uma doença respiratória grave, cuja transmissão se dá de pessoa para pessoa, através das gotículas de saliva ou superfícies contaminadas, com tempo de incubação que varia de 2 a 14 dias. O diagnóstico efetivo, a quarentena e os tratamentos de suporte são essenciais para a cura dos pacientes, evitando a disseminação e mortes (Zhai et al, 2020; Guan et al, 2020, Lipsitch et al, 2020)

O novo coronavírus SARS-CoV-2, vem de uma linhagem de outros coronavírus e é considerado um betacoronavírus, descoberto através de pesquisa em amostras de células epiteliais de vias aéreas humanas de pacientes com pneumonia. É o sétimo membro da família de coronavírus que infecta humanos. Seus antecessores, SARS-CoV e MERS-CoV, possuem características semelhantes e que provocam um quadro respiratório grave. Segundo Perlman 2020, uma característica que a Síndrome Respiratória Aguda Grave (SARS-CoV) trouxe foi um importante papel na economia e na vida social, semelhante com a que se observa hoje com o novo coronavírus e suas mutações. Com relação à Síndrome Respiratória do Oriente Médio 
(MERS- CoV), foi identificada pela primeira vez em setembro de 2012, em um empresário da Arábia Saudita que foi a óbito por insuficiência respiratória aguda (Memish, 2013). Segundo Azhar (2014), após o estudo em um paciente que foi à óbito acometido com o MERS e que teve contato com dromedário que apresentava rinorréia, realizou-se swabs nasais do paciente e do animal, ambos foram positivos para RNA MERS-CoV. O sequenciamento completo do genoma dos dois isolados foram idênticos.

Nos estudos de Schoeman \& Fielding (2019) e Almazan et al (2014), descreveram que os coronavírus que infectavam pássaros e mamíferos, nas últimas décadas se tornaram capazes de infectar humanos. O surto em 2003 do SARS (síndrome respiratória aguda grave) e em 2012, a síndrome respiratória do oriente médio (MERS), já comentado por outros autores, demonstrou que os coronavírus são capazes de cruzar barreiras entre as espécies e infectar humanos, provocando doenças respiratórias, entéricas, hepáticas e do sistema nervoso central, capazes de conduzir ao óbito, como o observado pelo novo coronavírus.

No Brasil, a introdução da COVID-19 ocorreu no mês de fevereiro, e foi declarado estado de Emergência de Saúde Pública de Importância Nacional (ESPIN), com implementação de diversas medidas sanitárias para conter o avanço da doença (Cavalcante et al, 2020). Os autores realizaram um estudo ecológico descritivo, analisando o Brasil, suas macrorregiões, Unidades Federativas, além de mais nove países com maior número de casos, concluindo que o Brasil estava entre os países com maiores números de casos e óbitos confirmados, com diferenças regionais.

Desde janeiro de 2020, a Organização Pan-Americana da Saúde (OPAS) vem apoiando as ações do Ministério da Saúde. De modo a promover o controle da COVID-19, nos estados com maior risco, a OPAS desenvolveu uma série de missões para troca de experiências no âmbito da vigilância, atenção à saúde e comunicação de risco. Entre os estados considerados da faixa vermelha até o dia 15 de março de 2021, receberam apoio da OPAS os estados de Goiás, Maranhão, Minas Gerais, Pará, Rio Grande do Norte e em outubro, a OPAS auxiliou o estado do Amapá a estruturar um Centro de Operações de Emergência.

Mesmo com todas as estratégias montadas para o controle da pandemia, não ocorreu uma regressão satisfatória no número de casos, voltando a valores alarmantes após a primeira onda. Diante do exposto, existe a necessidade de um olhar que identifique os momentos de maior disseminação e auxilie na busca de formas de controle e sensibilização das pessoas. Desta forma, este estudo, teve o objetivo de analisar o perfil de desenvolvimento da COVID-19 nos estados de maior risco de adoecimento até 15 de março de 2021, além de verificar quais os municípios de maior incidência de casos de COVID-19 dentre os estados de maior risco, quantificar os óbitos e calcular a taxa de letalidade e mortalidade nessas unidades federativas.

\section{Metodologia}

Trata-se de um estudo observacional-ecológico, descritivo, retrospectivo e de caráter epidemiológico. Os dados foram coletados do mapa de monitoramento de estados e capitais, (https://ciis.fmrp.usp.br/covid19/estado-br-mapa) e trabalhados individualmente, sendo selecionados os estados pertencentes à faixa vermelha de risco de adoecimento, na data de 15 de março de 2021. Para observar a disseminação e o quantitativo de casos, foi utilizado como ponto de corte os quatro primeiros municípios com maior número de casos, verificando-se a proporção de contribuição da doença naquele estado.

Realizou-se cálculo de mortalidade e letalidade por 100 mil habitantes dos 13 estados em foco. Para o tratamento descritivo dos dados, foi necessário observar estudos da dinâmica de transmissão, medidas de controle, informações sobre características demográficas, histórico de exposição, casos confirmados, número de óbitos e a disseminação da doença (Cavalcante et al 2020; Li et al 2020). Utilizou-se o software Microsoft Office Excel, 2020, para tratamento dos dados.

Uma vez que se trata de estudo com dados secundários, dispensa-se aprovação por meio de comitê de ética, seguindose às resoluções do Conselho Nacional de Ética em Pesquisa, para estudos com seres humanos no Brasil, nas normas 466/2012 
do Conselho Nacional de Saúde.

\section{Resultados}

No Brasil, até o dia 15 de março de 2021, observou-se um panorama de agravo em 13 estados, compondo uma linha vermelha, onde os gestores e cientistas concentram-se no combate à Pandemia. Dentre estes estados, a maior parte dos casos confirmados da COVID-19 concentrou-se na região Sudeste, e mais especialmente nos estados de São Paulo, com 2.208.242, e Minas Gerais, com 974.594, em um total de 3.182.836 casos confirmados. Em seguida, a maior contribuição veio da região Nordeste, destacando-se os estados do Maranhão, 228.827; Piauí, com 186.897; Ceará, com 474.978; Rio Grande do Norte, com 181.763; Paraíba, 239.594; e Alagoas, com 141.390, totalizando 1.453.449 casos. As demais regiões contribuíram com 717.588, no Centro Oeste, e 600.030, na região Norte. A região Sul encontra-se fora da linha vermelha de agravo (Figura 1).

Figura 1. Casos confirmados de COVID 19 nos estados brasileiros da faixa vermelha, até 15 de março de 2021.

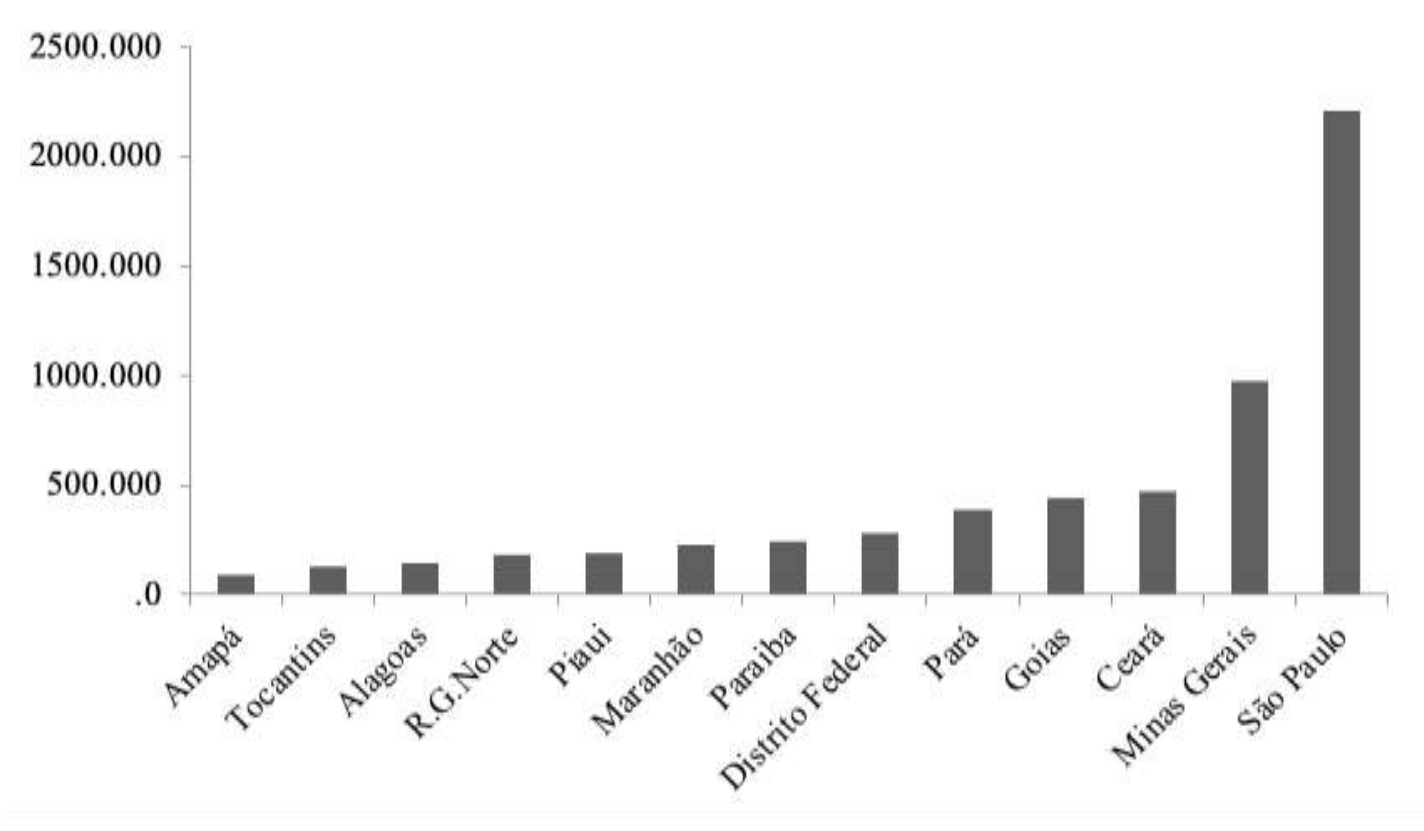

Fonte: Autores segundo Portal COVID-19 Brasil.

Na Tabela 1, observamos a distribuição proporcional dos casos confirmados nas quatro localidades de maior incidência da doença em cada estado. Observamos que 7 estados apresentaram mais de $40 \%$ dos casos confirmados nessas localidades. 
Tabela 1. Percentual de casos confirmados de COVID 19 em quatro municípios dos estados brasileiros, considerados na faixa vermelha de risco de adoecimento, até 15 de março de 2021.

\begin{tabular}{|c|c|c|c|c|}
\hline Região & Estado & Município & $\begin{array}{c}\text { Casos/Município } \\
(\%)\end{array}$ & $\begin{array}{l}\text { Total de quatro } \\
\text { municípios (\%) }\end{array}$ \\
\hline & & São Paulo capital & 25.3 & \\
\hline \multirow[t]{4}{*}{ Sudeste } & São Paulo & Campinas & 2.8 & 32.6 \\
\hline & & São Jose do Rio Preto & 2.3 & \\
\hline & & São José dos Campos & 2.2 & \\
\hline & & Belo Horizonte & 12,6 & \\
\hline \multirow[t]{4}{*}{ Sudeste } & Minas Gerais & Uberlândia & 7.6 & 24,7 \\
\hline & & Juiz de Fora & 2.2 & \\
\hline & & Contagem & 2.2 & \\
\hline & & São Luis & 13.9 & \\
\hline \multirow[t]{4}{*}{ Nordeste } & Maranhão & Imperatriz & 5.1 & 25,2 \\
\hline & & Balsas & 3.3 & \\
\hline & & Caxias & 2.8 & \\
\hline & & Teresina & 35.7 & \\
\hline \multirow[t]{4}{*}{ Nordeste } & Piauí & Parnaiba & 5.2 & 47.1 \\
\hline & & Floriano & 3.1 & \\
\hline & & Picos & 3.0 & \\
\hline & & Fortaleza & 29,3 & \\
\hline \multirow[t]{5}{*}{ Nordeste } & Ceará & Juazeiro do Norte & 3.94 & 39,5 \\
\hline & & Sobral & 3.43 & \\
\hline & & Caucaia & 2.84 & \\
\hline & & Natal & 28 & \\
\hline & & Parnamirim & 9,3 & 50,9 \\
\hline \multirow[t]{4}{*}{ Nordeste } & R. G. do Norte & Mossoró & 8,9 & \\
\hline & & Caicó & 4,6 & \\
\hline & & João Pessoa & 26.9 & \\
\hline & & Campina Grande & 9,2 & 42,6 \\
\hline \multirow[t]{3}{*}{ Nordeste } & Paraiba & Patos & 3,9 & \\
\hline & & Guarabira & 2,5 & \\
\hline & & Maceió & 39 & \\
\hline \multirow[t]{5}{*}{ Nordeste } & Alagoas & Arapiraca & 12,6 & \\
\hline & & Marechal Deodoro & 2,8 & 56,6 \\
\hline & & Santana do Ipanema & 2,1 & \\
\hline & & Goiânia & 26.1 & \\
\hline & & Aparecida de Goiânia & 11.4 & \\
\hline \multirow[t]{3}{*}{ Centro Oeste } & Goiás & Anápolis & 5,2 & 46,9 \\
\hline & & Rio Verde & 4,1 & \\
\hline & & Belém & 20.4 & \\
\hline \multirow[t]{4}{*}{ Norte } & Pará & Parauapebas & 9,2 & 37,6 \\
\hline & & Santarém & 4.0 & \\
\hline & & Ananindeua & 3.9 & \\
\hline & & Palmas & 25,7 & \\
\hline \multirow[t]{4}{*}{ Norte } & Tocantins & Araguaina & 17,8 & 53,4 \\
\hline & & Gurupi & 5,6 & \\
\hline & & Colinas do Tocantins & 4,3 & \\
\hline & & Macapá & 43,4 & \\
\hline \multirow[t]{3}{*}{ Norte } & Amapá & Santana & 22,3 & 78,8 \\
\hline & & Laranjal do Jari & 7,8 & \\
\hline & & Oiapoque & 5.2 & \\
\hline
\end{tabular}

Fonte: Autores segundo Portal COVID-19 Brasil.

Na Figura 2, está ilustrado os 12 estados da federação classificados na faixa vermelha, ou seja, com risco de adoecimento pela COVID-19. O Distrito Federal forneceu apenas os dados da cidade de Brasília, inviabilizando a quantificação dos dados de incidência das 4 localidades com maior número de casos. Destes, foram elencados 7 estados e seus respectivos municípios, que somados seus percentuais, demonstraram estar acima de $40 \%$ de risco para o adoecimento pela 
doença. Observa-se ainda que, quatro estados são pertencentes a região Nordeste, dois são do Norte e um é da região CentroOeste do Brasil (Tabela 1).

Figura 2. Percentual de casos confirmados de covid 19 nos estados brasileiros considerados na faixa vermelha de risco de adoecimento, até 15 de março de 2021.

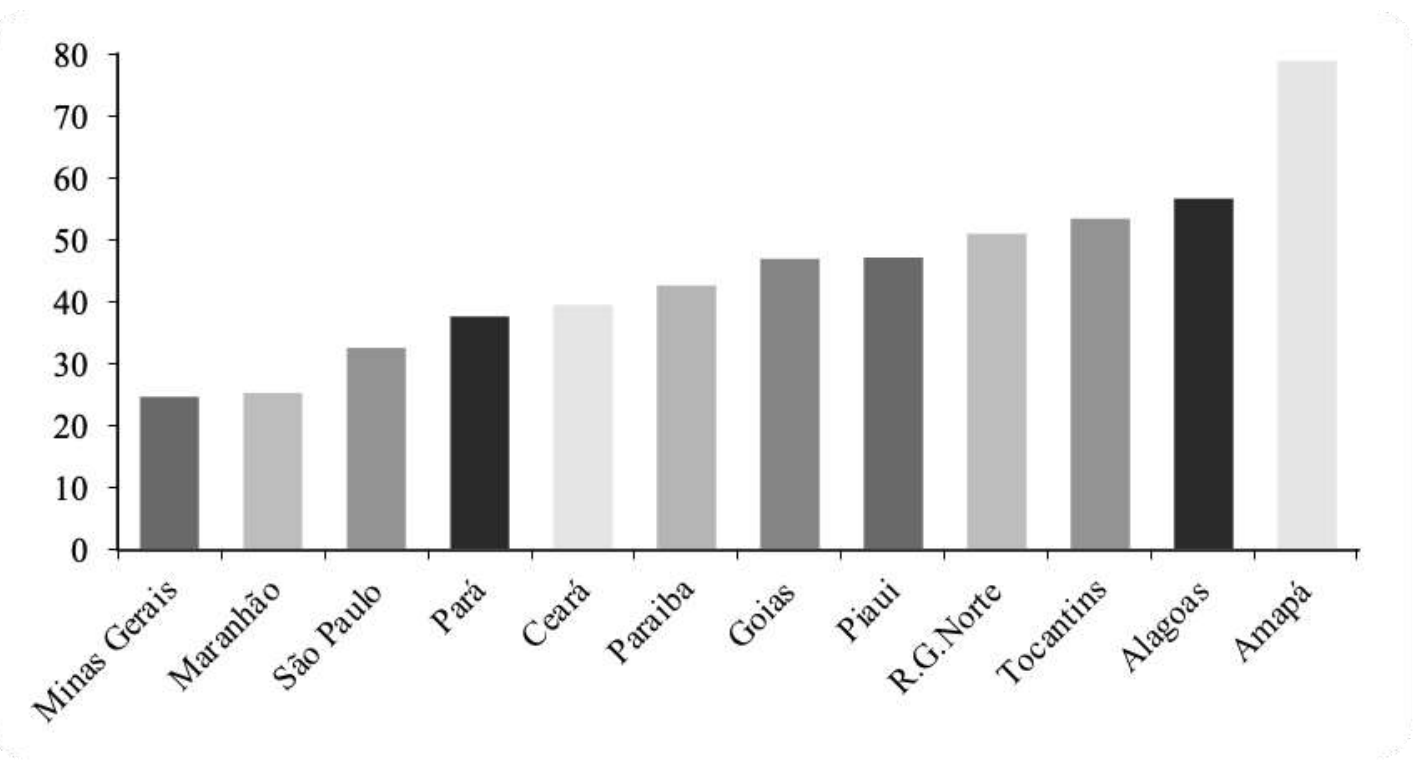

Fonte: Autores segundo portal covid-19 Brasil.

Nos estados em que houve maior número de casos confirmados da COVID-19 concentrados em poucos municípios, existe a possibilidade de bloqueio da referida patologia, através de ações educativas mais pontuais. Entretanto, quando se observa aqueles estados com a disseminação do vírus em vários municípios, a tendência de descontrole do surto é evidente. Nestes casos, demanda-se um maior contingente de profissionais, multiprofissionais e de outros seguimentos, tais como: saúde, segurança, serviços gerais, educação e serviços de apoio aos sepultamentos.

A Tabela 2, ilustra o número de casos confirmados, óbitos, letalidade e mortalidade pela COVID 19 nos 13 estados considerados na faixa vermelha. Os casos confirmados variaram, no estado do Amapá esteve entre 88.363 a 2.208 .242 casos de infecção pela COVID-19, e entre 1.187 a 64.223 óbitos; A taxa de letalidade entre Amapá, Tocantins e São Paulo, variou de 1.34 a 2.9, enquanto que a mortalidade foi de 0,07 a 1,08 entre o estado do Maranhão e Pará respectivamente. 
Tabela 2. Distribuição da letalidade e mortalidade por COVID-19, em 13 estados brasileiros, até 15 de março, 2021.

\begin{tabular}{cccccc}
\hline Estado & População & $\begin{array}{c}\text { Casos } \\
\text { Confirmados }\end{array}$ & Óbitos & Taxa Letalidade & $\begin{array}{c}\text { Taxa } \\
\text { Mortalidade }\end{array}$ \\
\hline Amapá & 829.424 & 88.363 & 1.187 & 1.34 & 0.14 \\
Pará & 8690.745 & 385.536 & 9.427 & 2.44 & 1.08 \\
Tocantins & 1590.248 & 126.131 & 1.697 & 1.34 & 1.06 \\
Maranhão & 7.114 .598 & 228.827 & 5.505 & 2.40 & 0.07 \\
Piaú́ & 3.281 .480 & 186.897 & 3.674 & 1.96 & 0.11 \\
Ceará & 9.187 .103 & 474.978 & 12.355 & 2.6 & 0.13 \\
Rio Grande do & 3.534 .165 & 181.763 & 3.937 & 2.16 & 0.11 \\
Norte & 4.039 .277 & 239.594 & 4.992 & 2.08 & 0.12 \\
Paraíba & 3.351 .543 & 141.390 & 3.216 & 2.27 & 0.09 \\
Alagoas & 7.113 .540 & 439.021 & 9.722 & 2.21 & 0.13 \\
Goiás & 3.055 .149 & 278.567 & 4.660 & 1.67 & 0.15 \\
Distrito Federal & 21.292 .666 & 974.594 & 20.687 & 2.12 & 0.09 \\
Minas Gerais & 46.289 .333 & 2.208 .242 & 64.223 & 2.9 & 0.13 \\
São Paulo & & & & & \\
\hline
\end{tabular}

Fonte: Autores segundo Portal COVID-19 Brasil.

Levando-se em consideração todas as regiões brasileiras, observamos que apenas a Região Sul, encontrava-se fora da faixa vermelha até 15 de março de 2021. Contudo, a Região Nordeste apresentou maior número de estados na faixa vermelha (6 estados), seguidos da Região Norte (3 estados). Chama a atenção o estado do Ceará, com taxa de mortalidade semelhante a de São Paulo, apresentando a segunda maior taxa de letalidade, em comparação aos 13 estados da faixa vermelha.

Ao analisar as taxas entre os 13 estados, observa-se que apesar das diferenças populacionais, como por exemplo, São Paulo e Amapá, a taxa de mortalidade entre os dois foi semelhante, 0,13/100.000 (São Paulo) e 0,14/100.000 mil habitantes (Amapá), representando magnitude similar da doença em diferentes níveis populacionais. A taxa de letalidade, entretanto, ao se comparar com os mesmos estados, observou-se uma maior diferença, Amapá 1,34 e São Paulo 2,9, possivelmente representando maior de risco de mortes entre as pessoas infectadas pela COVID 19.

\section{Discussão}

A atenção ao descontrole deve ser priorizada com orientações como as do estudo de Perlman 2020, em que medidas de saúde pública, com inclusão de quarentena, diagnóstico preciso, adesão da comunidade às orientações de prevenção e estratégias bem conduzidas nos ambientes de saúde, foram fundamentais no controle da SARS-CoV e MERS-CoV. Da mesma forma, Houshue et al (2020), destaca a importância de sintonia entre a equipe de saúde e as autoridades de saúde pública nas três esferas de governo (municipal, estadual e federal), além de informações clínicas, rápidas e precisas, com relação à atendimentos de pacientes.

No estudo de Li et al (2020), nos primeiros 425 pacientes, a idade média dos casos positivos foi de 59 anos, $56 \%$ do sexo masculino, 55 \% estavam ligados ao Mercado Atacadista de frutos do Mar de Huanan, o período de incubação foi de 5,2 dias (Intervalo de Confiança de 95\% [IC] 4,1 a 7,0), detectaram que nos estágios iniciais a epidemia duplicava a cada 7,4 dias, que o contato era de pessoa a pessoa, quando muito próximas. Logo, a disseminação é rápida e não há como evitá-la sem as medidas de distanciamento e proteção individual.

Almeida et al (2020), descreveu sobre as mudanças nas condições socioeconômicas e de saúde dos brasileiros durante 
o período de pandemia de COVID-19, em um estudo transversal de comportamentos pela internet, durante um mês, de 24 de abril a 24 de maio de 2020. Participaram 45.161 pessoas recrutadas por amostragem em cadeia e os achados conclusivos mostraram a importância do controle da pandemia no Brasil, para que os efeitos deletérios da situação socioeconômica e de saúde relacionados às medidas de restrição social, não se perpetuem e tragam piores consequências.

É importante observar o ocorrido durante o surgimento de coronavírus anteriores, como SARS e MERS. No estudo de Xie and Chen (2020) e Zou et al (2020) ressalta-se a necessidade de medidas e decisões terapêuticas utilizadas para o controle da Síndrome Respiratória Aguda Grave causada pelo SARS-CoV e Síndrome Respiratória do Oriente Médio causada pelo MERS-CoV, observando o crescimento exponencial dos casos no início de 2020. Dessa forma, associando experiências adquiridas no cenário pandêmico atual às lições durante o aparecimento de outros coronavírus, pode-se sugerir planejamentos de controle atualmente e no futuro.

O novo coronavírus sofre mutações, alertando mais ainda, que a comunidade cientifica e autoridades em saúde devem priorizar o investimento em pesquisas, principalmente na genética e formulações de vacinas, evitando o estrangulamento do sistema de saúde, que não comporta o grande número de pacientes graves (UTIs) ou na atenção básica. Vários estudos relatam a disseminação através de viagens de negócios nacionais e internacionais, além de trabalhos em locais que comportam grande números de pessoas e serviços de transportes de passageiros terrestres e aéreos (Zhu et al 2019; Pogpuril et al 2020; Liu et al 2020; Zou et al 2020).

\section{Conclusão}

Neste estudo, foi destacado um pequeno recorte de determinado período, 15 março de 2021, momento em que 13 estados brasileiros estavam numa situação de risco para COVID-19 e que em sete desses estados, mais de $40 \%$ dos casos positivos se concentravam em apenas 04 municípios, sendo possível, realizar um trabalho de contenção da doença, com medidas mais efetivas, já realizadas, mas que precisam ser melhoradas, através de orientações mais próximas da realidade do povo brasileiro. A pandemia atinge locais distantes com realidades distintas, tais como serviços de saúde, padrão cultural e socioeconômico, os quais impactam nos resultados das estratégias implementadas.

Dessa forma, a distribuição de casos confirmados, atingindo um maior número de municípios, aumenta a probabilidade de disseminação da COVID 19, o que torna a situação ainda mais alarmante. De modo que os danos possam ser minimizados, e possa haver uma contenção eficaz com diminuição das taxas de transmissibilidades, pode-se elencar algumas medidas: 1) a ratificação da Educação para a Saúde específica para a COVID-19, com aproximação ao cotidiano da população, com equipes da Estratégia de Saúde da Família atuando ostensivamente nos núcleos familiares; 2) a manutenção das medidas de segurança sanitária, como o uso de máscara, com obrigatoriedade de uso em todos os lugares; 3) a qualificação e posterior participação dos educadores do ensino público e privado, no comprometimento pela educação sobre a COVID-19, às famílias de seus alunos; 4) nos municípios onde os casos confirmados estiverem acima de 40\%, sejam realizadas barreiras sanitárias nas rodovias, para evitar a disseminação para os demais municípios; 5) adoção do uso da inteligência local em saúde, que combina o rastreamento de contatos e testes rápidos para mitigar surtos de COVID-19, por todos os gestores municipais.

Naturalmente, é elementar que as demais estratégias já em andamento, permaneçam e sejam fortalecidas. As avaliações periódicas do quadro epidemiológico, devem também conter os dados de casos confirmados, óbitos, internações, e recuperados, para que as estratégias implementadas continuem ou sejam aperfeiçoadas.

\section{Referências}

Almazán, F., Sola, I., Zuñiga, S., Marquez-Jurado, S., Morales, L., Becares, M., \& Enjuanes, L. (2014). Coronavirus reverse genetic systems: Infectious clones and replicons. Virus Research, 189, 262-270. https://doi.org/10.1016/j.virusres.2014.05.026 
Azhar, E. I., El-Kafrawy, S. A., Farraj, S. A., Hassan, A. M., Al-Saeed, M. S., Hashem, A. M., \& Madani, T. A. (2014). Evidence for Camel-to-Human Transmission of MERS Coronavirus. New England Journal of Medicine, 370(26), 2499-2505. https://doi.org/10.1056/nejmoa1401505

Cavalcante, J. R., Cardoso-Dos-Santos, A. C., Bremm, J. M., Lobo, A. de P., Macário, E. M., Oliveira, W. K. de, \& França, G. V. A. de. (2020). COVID-19 no Brasil: evolução da epidemia até a semana epidemiológica 20 de 2020. Epidemiologia e Servicos de Saude : Revista Do Sistema Unico de Saude Do Brasil, 29(4), e2020376. https://doi.org/10.5123/s1679-49742020000400010.

COVID-19 Brasil (2021). Monitoramento - Estados e capitais: Mapa. https://ciis.fmrp.usp.br/covid19/estado-br-mapa. Recuperado em 13 de Março de 2021.

de Almeida, W. da S., Szwarcwald, C. L., Malta, D. C., Barros, M. B. de A., Júnior, P. R. B. de S., Azevedo, L. O., Romero, D., Lima, M. G., Damacena, G. N., Machado, Í. E., Gomes, C. S., de Pina, M. de F., Gracie, R., Werneck, A. O., \& da Silva, D. R. P. (2020). Changes in brazilians' socioeconomic and health conditions during the covid-19 pandemic. Revista Brasileira de Epidemiologia, 23, 1-14. https://doi.org/10.1590/1980-549720200105

Guan, W., Ni, Z., Hu, Y., Liang, W., Ou, C., He, J., Liu, L., Shan, H., Lei, C., Hui, D. S. C., Du, B., Li, L., Zeng, G., Yuen, K.-Y., Chen, R., Tang, C., Wang, T., Chen, P., Xiang, J., ... Zhong, N. (2020). Clinical Characteristics of Coronavirus Disease 2019 in China. New England Journal of Medicine, 382(18), 1708-1720. https://doi.org/10.1056/nejmoa2002032

Holshue, M. L., DeBolt, C., Lindquist, S., Lofy, K. H., Wiesman, J., Bruce, H., Spitters, C., Ericson, K., Wilkerson, S., Tural, A., Diaz, G., Cohn, A., Fox, L., Patel, A., Gerber, S. I., Kim, L., Tong, S., Lu, X., Lindstrom, S., ... Pillai, S. K. (2020). First Case of 2019 Novel Coronavirus in the United States. New England Journal of Medicine, 382(10), 929-936. https://doi.org/10.1056/nejmoa2001191.

Instituto Brasileiro de Geografia e Estatística (2020). IBGE Cidades e Estados. https://cidades.ibge.gov.br/. Recuperado em 15 de Março de 2021.

Koven, S. (2020). They Call Us and We Go. New England Journal of Medicine, 382(21), 1978-1979. https://doi.org/10.1056/nejmp2009027

Li, Q., Guan, X., Wu, P., Wang, X., Zhou, L., Tong, Y., Ren, R., Leung, K. S. M., Lau, E. H. Y., Wong, J. Y., Xing, X., Xiang, N., Wu, Y., Li, C., Chen, Q., Li, D., Liu, T., Zhao, J., Liu, M., ... Feng, Z. (2020). Early Transmission Dynamics in Wuhan, China, of Novel Coronavirus-Infected Pneumonia. New England Journal of Medicine, 382(13), 1199-1207. https://doi.org/10.1056/nejmoa2001316

Lipsitch, M., Swerdlow, D. L., \& Finelli, L. (2020). Defining the Epidemiology of Covid-19 — Studies Needed. New England Journal of Medicine, 382(13), 1194-1196. https://doi.org/10.1056/nejmp2002125

Memish, Z. A., Zumla, A. I., Al-Hakeem, R. F., Al-Rabeeah, A. A., \& Stephens, G. M. (2013). Family Cluster of Middle East Respiratory Syndrome Coronavirus Infections. New England Journal of Medicine, 368(26), 2487-2494. https://doi.org/10.1056/nejmoa1303729

Organização Pan-Americana de Saúde - OPAS (2021). https://www.paho.org/pt/brasil. Recuperado em 13 de Março de 2021

Perlman, S. (2020). Another Decade, Another Coronavirus. New England Journal of Medicine, 382(8), 760-762. https://doi.org/10.1056/nejme1917479

PRODAP - Centro de Gestão da Tecnologia da Informação - Governo do Amapá (2021). Coronavírus. https://corona.portal.ap.gov.br.

Schoeman, D., Fielding, B. C., Arias-Reyes, C., Zubieta-DeUrioste, N., Poma-Machicao, L., Aliaga-Raudan, F., Carvajal-Rodriguez, F., Dutschmann, M., Schneider-Gasser, E. M., Zubieta-Calleja, G., Soliz, J., Schneider-Gasser, E. M., Zubieta-Calleja Director High, G., Loeffelholz, M. J., Tang, Y. W., Velavan, T. P., Meyer, C. G., Taylor, D., Lindsay, A. C., ... Sheraton, K. (2020). Journal Pre-proof Does the pathogenesis of SAR-CoV-2 virus decrease at highaltitude? Does the pathogenesis of SAR-CoV-2 virus decrease at high-altitude? Corresponding authors. Cell Research, 9(1), 278-280. https://doi.org/10.3390/ijerph17082932

Xie, M., \& Chen, Q. (2020). Insight into 2019 novel coronavirus - An updated interim review and lessons from SARS-CoV and MERS-CoV. International Journal of Infectious Diseases, 94, 119-124. https://doi.org/10.1016/j.ijid.2020.03.071

Zhai, P., Ding, Y., Wu, X., Long, J., Zhong, Y., \& Li, Y. (2020). The epidemiology, diagnosis and treatment of COVID-19. International Journal of Antimicrobial Agents, 55(5). https://doi.org/10.1016/j.ijantimicag.2020.105955

Zhu, N., Zhang, D., Wang, W., Li, X., Yang, B., Song, J., Zhao, X., Huang, B., Shi, W., Lu, R., Niu, P., Zhan, F., Ma, X., Wang, D., Xu, W., Wu, G., Gao, G. F., \& Tan, W. (2020). A Novel Coronavirus from Patients with Pneumonia in China, 2019. New England Journal of Medicine, 382(8), 727-733. https://doi.org/10.1056/nejmoa2001017

Zou, L., Ruan, F., Huang, M., Liang, L., Huang, H., Hong, Z., Yu, J., Xia, J., Guo, Q., Hui-Ling Yen, \& Wu, J. (2020). SARS-CoV-2 Viral Load in Upper Respiratory Specimens of Infected Patients. New England Journal of Medicine, 382(12), 1175-1177. https://doi.org/10.1056/NEJMc2001737 